\title{
THE ITERATION OF POLYNOMIALS AND TRANSCENDENTAL ENTIRE FUNCTIONS
}

\author{
I. N. BAKER \\ (Received 23 May 1979; revised 1 May 1980) \\ Communicated by E. Strzelecki
}

\begin{abstract}
The iterative behaviour of polynomials is contrasted with that of small transcendental functions as regards the existence of unbounded domains of normality for the sequence of iterates.
\end{abstract}

1980 Mathematics subject classification (Amer. Math. Soc.): 30 D 05.

\section{Introduction}

If $f$ is a rational or entire function of the complex variable $z$ its natural iterates $f^{n}$ are defined by $f^{1}(z)=f(z), f^{n+1}(z)=f\left\{f^{n}(z)\right\}, n=1,2, \ldots$ The theory devel-

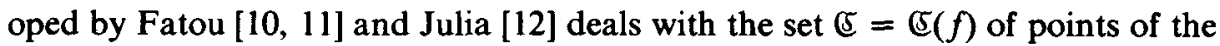
complex plane in whose neighbourhood $\left(f^{n}\right)$ is a normal family. It is convenient to express many results in terms of the complement $\mathfrak{F}(f)$ of $\mathbb{E}$, that is the set of non-normality. We shall assume throughout that $f$ is not a rational function of order 0 or 1 . Then $\mathfrak{F}(f)$ has the following properties (see [10] and [11]):

I. $\mathfrak{F}(f)$ is a non-empty perfect set.

II. $\mathfrak{F}(f)$ and $\mathfrak{E}(f)$ are completely invariant under the mapping $z \rightarrow f(z)$.

In general a set $S$ is called invariant under $z \rightarrow f(z)$ if $\alpha \in S$ implies $f(\alpha) \in S$. The set $S$ is completely invariant if $\alpha \in S$ implies both $f(\alpha) \in S$ and $\beta \in S$ for every solution $\beta$ of $f(\beta)=\alpha$.

While extensive and detailed results have been obtained for rational $f$, work on the transcendental entire case is restricted to showing that certain of the main 
results extend from the rational case (for example [1], [3], [11]) with a few discussions of examples and of features unique to the entire functions. Common to both classes are the polynomials, assumed to have degree at least two, for which it is obvious that $f^{n}(z) \rightarrow \infty$ in a neighbourhood of $\infty$. Indeed it follows from II that:

III. If $f$ is a polynomial of degree at least two then $\Subset(f)$ contains a neighbourhood of $\infty$. Thus there is just one unbounded component $D$ of $\Subset(f)$ and $D=\{z$ : $\left.f^{n}(z) \rightarrow \infty\right\}$. Further $D$ is invariant and completely invariant.

It is natural to examine the effect on the assertions of III of replacing $f$ by a transcendental entire function. It is obvious from I and Picard's theorem that $\mathfrak{F}$ is unbounded in this case, so that $\mathcal{E}(f)$ no longer forms a neighbourhood of $\infty$. It will be shown that if $f$ is transcendental but of sufficiently small growth $\S(f)$ can have no unbounded completely invariant component and under suitable assumptions no unbounded component at all:

THEOREM 1. If for transcendental entire $f$ there is an unbounded invariant component of $\&(f)$, then the growth of $f$ must exceed order $\frac{1}{2}$, minimal type.

THEOREM 2. If the maximum modulus $M(r, f)$ of the transcendental entire function $f$ satisfies

$$
\log M(r, f)=O\left\{(\log r)^{p}\right\} \text { as } r \rightarrow \infty,
$$

where $1<p<3$, then every component of $\mathfrak{S}(f)$ is bounded.

The order of growth in Theorem 1 is sharp as we shall prove by consideration of $\cos \left\{\left(\varepsilon^{2} z+\frac{9}{4} \pi^{2}\right)^{1 / 2}\right\}$ for any $\varepsilon$ such that $0<\varepsilon<(3 \pi)^{1 / 2}$. The sharpness or otherwise of Theorem 2 remains open, although the proof given does not seem to extend to functions of larger growth.

Since for transcendental $f$ any completely invariant component of $\mathbb{E}(f)$ is unbounded and invariant Theorem 1 has the corollary:

THEOREM 3. If $f$ is transcendental and entire of growth not exceeding order $\frac{1}{2}$ minimal type, then $\mathbb{S}(f)$ has no completely invariant component.

This complements the results of [2], where it was shown that a polynomial may, like $z^{2}$, have two completely invariant components of $\varepsilon(f)$, but in the case of transcendental $f$ at most one such component can occur. The example $\cos \left\{\left(\varepsilon^{2} z+\frac{9}{4} \pi^{2}\right)^{1 / 2}\right\}$ shows that the theorem is sharp.

In the course of the proofs we have to discuss the possible limit functions of subsequences of $\left(f^{n}\right)$ in the domains concerned. The necessary results are 
developed in Section 2 and include

THEOREM 4. Let $D$ be a domain of the complex plane with at least three boundary points and let $f$ be analytic in $\bar{D}$, except that if $D$ is unbounded $f$ need not be analytic at $\infty$. Let $f$ map $D$ into itself and suppose that no subsequence of $f^{n}$ has limit $z$ in $D$ (in particular this is so if $f$ is not a univalent map of $D$ onto $D$ ). Then the whole sequence $\left(f^{n}\right)$ converges in $D$ to a constant limit $\alpha \in \bar{D}$.

Specializing to entire functions one has

TheOrEM 5. If $f$ is entire and non-linear and $D$ is an invariant component of $\S(f)$, then either (i) $f$ is a univalent map of $D$ onto itself such that $f_{k}^{n_{k}}(z) \rightarrow z$ in $D$ for some $n_{k}$ such that $n_{k} \rightarrow \infty$, or (ii) the whole sequence $\left(f^{n}\right)$ converges in $D$ to a constant limit which belongs to $\bar{D}$. In particular every limit function is finite except in the case when $f^{n}(z) \rightarrow \infty$ in $D$. Further, if $f$ is transcendental then $D$ is simply-connected.

Both cases (i) and (ii) can occur: (i) occurs precisely when the domain $D$ contains a centrum, that is a fixed point $\alpha, f(\alpha)=\alpha$, such that $\left|f^{\prime}(\alpha)\right|=1$. Since $\left(f^{n}\right)$ is a normal family near $\alpha$ it turns out that $f^{\prime}(\alpha)=\exp (i \pi \theta)$, where $\theta$ is real and irrational, and indeed there are further restrictions on $\theta$. See for example [8] and [15].

Theorems 4 and 5 are analogous to results of Wolff [17] and Denjoy [9], who derive similar consequences for general analytic maps of the open unit disc into itself. The results do not carry over directly to our case by conformal mapping even for simply connected $D$, because the boundary may be extremely wild.

A further auxiliary result seems to be of some independent interest:

TheOREM 6. Let $f$ be entire and let $D$ be an unbounded invariant component of $\Im(f)$ in which $f^{n}(z) \rightarrow \infty$. Then there exists a path approaching $\infty$ in $D$ on which $|f(z)|=O\left(|z|^{k}\right)$ for some constant $k$.

This gives an extension in some respects of results of Bhattacharyya [6], [7], who obtained the same conclusion without the hypothesis that $f^{n}(z) \rightarrow \infty$ but assuming that $D$ contains an angle. Bhattacharyya also has some special cases, now contained in Theorem 1, about the existence of unbounded invariant domains of particular types. 


\section{Proof of Theorems 4 and 5}

LeMma 1. Let $D$ be a domain with at least three boundary points and let $f$ be an analytic function such that $f(D) \subset D$. If some subsequence of $\left(f^{n}\right)$ has a non-constant limit function in $D$, then there is a subsequence of $\left(f^{n}\right)$ which converges to $z$ in $D$.

ProOF. $\left(f^{n}\right)$ is normal in $D$. Suppose that $f^{n_{i}}$ has non-constant limit $g$ in $D$. The values of $g(z)$ are in $\bar{D}$ and indeed, by Hurwitz' Theorem, in $D$.

Put $p_{i}=f^{n_{i}}, q_{i}=f^{n_{i}-n_{i-1}}$, where we may assume that $n_{i}-n_{i-1} \rightarrow \infty$. Then $q_{i}\left(p_{i-1}\right)=p_{i}$. There is a subsequence of $q_{i}$ which converges in $D$ to a limit $h$ and we have $h(g)=g$ in $D$, which implies $h(z) \equiv z$.

We remark further that in the circumstances of Lemma $1, f$ is a homeomorphism of $D$ onto $D$. For if $f^{n_{i}-n_{i-1}}(z) \rightarrow z$ then $f$ must be univalent in $D$. Also if $f$ omits a value $z_{0}$ of $D$ then so do all $f^{n}$ and thus $h=\lim f^{n_{i}-n_{j-1}}$ either omits $z_{0}$ or is identically the constant $z_{0}$, neither of which is admissible. Hence we have

LeMmA 2. Let $D$ be as in Lemma 1. If $f$ is an analytic map of $D$ into itself but is not a univalent map of $D$ onto $D$, then every convergent subsequence of $\left(f^{n}\right)$ has a constant limit.

If $z_{0}=\lim f^{n_{1}}(z)$ in $D$ and if $f$ is continuous at $z_{0}$ then $f\left(z_{0}\right)=z_{0}$.

To prove the last part observe that

$$
f\left(z_{0}\right)=f\left\{\lim f^{n_{i}}(z)\right\}=\lim f\left\{f^{n_{i}}(z)\right\}=\lim f^{n_{i}}\{f(z)\}=z_{0} .
$$

Proof of TheOREM 4. The assumptions assure that only constant limit functions occur by Lemma 1. Take any $z_{0} \in D$ and let $D_{0}$ be a relatively compact subdomain of $D$ which contains both $z_{0}$ and $f\left(z_{0}\right)$. Define $D_{n}=f^{n}\left(D_{0}\right)$; then $D_{n-1}$ meets $D_{n}$ for each $n$ and so $A_{n}=\bigcup_{k=n}^{\infty} D_{k}$ is connected for each $n$.

Note that $\lim \sup A_{n}=\{x$; each neighbourhood of $x$ meets infinitely many $\left.A_{n}\right\}=\left\{x\right.$; each neighbourhood of $x$ meets infinitely many $\left.D_{n}\right\}$ is identical with the set of the (constant) limit functions of subsequences of $\left(f^{n}\right)$ in $D_{0}$ (and hence in $D$ ). Further since $A_{n}$ is a monotone decreasing family of sets, $L=\lim \sup A_{n}$ is identical with $\lim \inf A_{n}=\{x$; each neighbourhood of $x$ meets all but finitely many $\left.A_{n}\right\}$. Thus on the compact space $S=\mathrm{C} \cup\{\infty\}$ the connected sets $A_{n}$ have a non-empty $\lim \inf A_{n}$ and (see for example [16, p. 14]) $L$ is connected. $L$ is also closed and thus a continuum or point in $S$.

If $L$ contains more than one point it is uncountable. By Lemma 2 each point $z_{0}$ of $L$ (except perhaps $z_{0}=\infty$ ) satisfies $f\left(z_{0}\right)=z_{0}$. Since $f$ is analytic in $\bar{D}$ and $L \subset \bar{D}$ the uncountability of $L$ would imply that $f(z) \equiv z$, and so $f^{n}(z) \rightarrow z$, against the assumptions. Hence $L$ consists of a single point and the result follows. 
LEMMA 3 ([11]). If $f$ is non-linear and entire, $\beta \in \mathfrak{F}(f), U$ is a neighbourhood of $\beta$ and $K$ is an arbitrary compact plane set which omits the Picard exceptional value of $f$, if such exists then there exists a positive integer $n_{0}$ such that for all $n>n_{0}$ we have

$$
f^{n}(U) \supset K
$$

This implies

LEMMA 4. If in any component $D$ of $₫(f)$ there is a sequence $f^{n_{1}}(z)$ which tends to a finite limit function, then $D$ is simply-connected.

Proof of Theorem 5. If $f$ is non-linear and entire any component $D$ of $\mathbb{E}(f)$ must have at least three boundary points. Since $f(D) \subset D$ for an invariant $D$, the assumptions of Theorem 4 apply and we must have one of the alternatives (i) or (ii).

If there is at least one finite limit function, Lemma 4 shows that $D$ is simply-connected. If not the whole sequence $f^{n}$ must converge to $\infty$ in $D$ so that $D$ is unbounded. But Theorem 1 of [4] states that any unbounded component of $(S(f)$ is simply-connected, provided that $f$ is transcendental.

It remains only to show that in case (i) every limit function is finite. Map $D$, which is certainly simply-connected, to $K=\{|w|<1\}$ by $w=\psi(z)$. Then $g=\psi \circ f \circ \psi^{-1}$ maps $K$ univalently onto $K$ and so is a Moebius transformation. From $f^{n_{1}}(z) \rightarrow z$ it follows that $g^{n_{3}}(w) \rightarrow w$. Examination of those Moebius transformations which map $K$ onto $K$ shows that this can happen only when $g$ is a non-euclidean rotation about a fixed point, which we can without loss of generality take to be at $w=0$. Thus $g(w)=e^{i \gamma} w$, where $\gamma$ is real, and $f=$ $\psi^{-1}\left(e^{i \gamma} \psi\right)$. Then for any $z$ in $D$ we have $\psi(z)$ in $K$ so $|\psi(z)|=\rho<1$, and $f^{n}(z)=\psi^{-1}\left(e^{i n \gamma} \psi(z)\right)$ shows that the values $f^{n}(z)$ all lie on the compact subset $\psi^{-1}\{w ;|w|=\rho\}$ of $D$. Every limit function of $f^{n}$ must then be finite.

REMARK. If $\alpha=\psi^{-1}(0)$ then $\alpha$ is the unique solution of $f(z)=z$ in $D$ and $f^{\prime}(\alpha)=e^{i \gamma}$. Obviously $\alpha \in D \subset \Subset(f)$. Direct computations (see for example [11]) show that if $\gamma$ is rational we cannot have $\alpha \in \mathbb{E}(f)$. Rüssmann [15] has shown that for almost all irrational $\gamma$ a fixed point with $f^{\prime}(\alpha)=e^{i \gamma \pi}$ does belong to $\subseteq(f)$, while Cremer [8] shows that for certain Liouville numbers this may not be the case. Thus we can ensure case (i) of Theorem 5 by making $f$ have a fixed point $\alpha$ with a suitably chosen $f^{\prime}(\alpha)=e^{i \gamma \pi}$, and $D$ will be the component of $\mathbb{E}(f)$ to which $\alpha$ belongs. Case (ii) may be realised similarly by making $f$ have an attrative fixed point $\alpha$ such that $f(\alpha)=\alpha,\left|f^{\prime}(\alpha)\right|<1$. 


\section{Proof of Theorem 6}

LEMMA 5. In a domain $D$ the analytic functions $g$ of the family $G$ omit the values $0,1 . K$ is a compact connected subset of $D$ on which the functions all satisfy $|g(z)| \geqslant 1$. Then there exist constants $B, C$ dependent only on $K$ and $D$ and such that for any $z, z^{\prime}$ in $K$ and any $g$ in $G$ we have

$$
\left|g\left(z^{\prime}\right)\right|<B|g(z)|^{C} .
$$

Proof. Denote by $4 \delta$ the distance of $K$ from the boundary of $D$. There is a finite collection $F$ of $N$ discs of radius $\delta$ whose centres lie on $K$ and whose union covers $K$. Since $K$ is connected there is for any pair $z, z^{\prime}$ in $K$ a chain of $p<N$ points $z=w_{1}, w_{2}, \ldots, w_{p}=z^{\prime}$ in $K$ such that $w_{i}, w_{i+1}$ lie in a common disc of $F$. Thus $\left|w_{i+1}-w_{i}\right|<2 \delta$.

In the unit disc $g\left(w_{i}+3 \delta z\right)$ is analytic and omits the values 0 , 1. Now Schottky's theorem (see for example [13]) states that there is an absolute constant $C^{\prime}$ such that for every function $f$ which is analytic and unequal to 0,1 in the unit disc we have for $0<r<1$

$$
\max _{|z|<r}|f(z)|<\exp \left[\frac{1}{1-r}\left((1+r) \log \max (1, S|f(0)|)+2 C^{\prime} r\right)\right] .
$$

Applying this to $g\left(w_{i}+3 \delta z\right)$ and noting $\left|g\left(w_{i}\right)\right| \geqslant 1$ we have

$$
\left|g\left(w_{i+1}\right)\right|<A\left|g\left(w_{i}\right)\right|^{5}
$$

for some absolute constant $A$. Hence

$$
\left|g\left(z^{\prime}\right)\right|<B|g(z)|^{C},
$$

where $C=5^{N}, B=A^{1+5+\cdots+5^{N}}$ are constants independent of the choice of $g$ or of $z, z^{\prime}$ in $K$.

Proof of Theorem 6. We may assume that $f$ is transcendental. Replacing $f(z)$ by $(f(a+b z)-a) / b, a, b$ constant, subjects $\mathfrak{F}(f)$, $\mathfrak{E}(f)$ to a linear transformation. Since $\mathfrak{F}$ is unbounded we may without loss of generality assume that 0 and 1 belong to $\mathfrak{F}$.

Take $z_{1}$ in the unbounded invariant component $D$ of the hypothesis. Then $z_{2}=f\left(z_{1}\right) \neq z_{1}$ since $f^{n}(z) \rightarrow \infty$ in $D$. Join $z_{1}$ and $z_{2}$ by a path $\gamma_{1}$ in $D$ and define $z_{n+1}=f\left(z_{n}\right), \gamma_{n+1}=f\left(\gamma_{n}\right)$ for $n=1,2, \ldots$, so that $\gamma_{n}$ is a path which joins $z_{n}$ to $z_{n+1}$ in $D$ and, moreover, $\gamma_{n} \rightarrow \infty$. Thus $\Gamma=\cup \gamma_{n}$ is a path which goes to $\infty$ in $D$.

Since $f^{n} \rightarrow \infty$ on $\gamma_{1}$, we have $\left|f^{n}(z)\right|>1$ on $\gamma_{1}$ for $n \geqslant n_{0}$ and $f^{n} \neq 0,1$ in $D$. Thus by Lemma 5 there are constants $B$ and $C$ such that

$$
\left|f^{n}\left(t^{\prime}\right)\right|<B\left|f^{n}(t)\right|^{C}, \quad t, t^{\prime} \in \gamma_{1}, n \geqslant n_{0} .
$$


In particular $t^{\prime}=z_{2}, t=z_{1}$ leads to $\left|z_{n+2}\right|<B\left|z_{n+1}\right|^{C}$. For any point $z$ sufficiently far out on $\Gamma$ we have $z \in \gamma_{n}$ for some $n \geqslant n_{0}+1$ and so $z=f^{n-1}(t)$ for some $t \in \gamma_{1}$. Then

$$
|z|=\left|f^{n-1}(t)\right|<B\left|f^{n-1}\left(z_{1}\right)\right|^{C}=B\left|z_{n}\right|^{C},
$$

and also $\left|z_{n+1}\right|=\left|f^{n-1}\left(z_{2}\right)\right|<B|z|^{C}$. Hence $f(z) \in \gamma_{n+1}$ satisfies

$$
|f(z)|<B\left|z_{n+1}\right|^{C}<B\left(B|z|^{C}\right)^{C}=B^{1+C}|z|^{C^{2}} \text {. }
$$

COROLlary. The situation of Theorem 6 can occur only if $f$ has growth of at least order $\frac{1}{2}$, mean type.

An example which shows the sharpness of the order of growth in the corollary is $f(z)=\sin \sqrt{z} / \sqrt{z}+z+a$ for sufficiently large positive $a$.

For the domain $D: x>a^{2}, y^{2}<4(x+1)$ where $z=x+i y$, is mapped by the principal branch of $w=u+i v=z^{1 / 2}$ into a subset of $|v|<1, u>a$. Thus

$$
\left|\frac{\sin \sqrt{z}}{\sqrt{z}}\right|<e|z|^{-1 / 2}, \quad z \in D,
$$

and so

$$
|f(z)-(z+a)|<e|z|^{-1 / 2}, \quad z \in D .
$$

If $z \in D$ let $z_{0}=x_{0}+i y_{0}$ be on the boundary of $D$. Either $x_{0}<x+\frac{1}{2} a$, in which case $\left|z+a-z_{0}\right| \geqslant \frac{1}{2} a$, or $x_{0} \geqslant x+\frac{1}{2} a$, in which case

$$
\left|y_{0}\right|=2\left(x_{0}+1\right)^{1 / 2} \geqslant 2\left(x+1+\frac{1}{2} a\right)^{1 / 2}, \quad|y|<2(x+1)^{1 / 2},
$$

so that

$$
\begin{aligned}
\left|z+a-z_{0}\right| & \geqslant\left|y_{0}\right|-|y| \\
& >2\left(x+1+\frac{1}{2} a\right)^{1 / 2}-2(x+1)^{1 / 2} \\
& >\frac{1}{2} a\left(x+1+\frac{1}{2} a\right)^{-1 / 2} .
\end{aligned}
$$

Thus in all cases the inequality

$$
\left|z+a-z_{0}\right|>\frac{1}{2} a\left(x+1+\frac{1}{2} a\right)^{-1 / 2}
$$

holds and the distance of $z+a$ from the boundary of $D$ is at least

$$
\frac{1}{2} a\left(x+1+\frac{1}{2} a\right)^{-1 / 2}>e|z|^{-1 / 2}
$$

for all $z \in D$ if $a$ is sufficiently large. For such a choice of $a, f(D) \subset D$. Thus $f^{n}$ is normal in $D$ and $D$ forms part of a component $D_{1}$ of $\subseteq(f)$, which satisfies $f\left(D_{1}\right) \subset D_{1}$. If $x_{0}$ is sufficiently large the segment $I=\left[x_{0}, \infty\right)$ of the real axis belongs to $D$ and has the property that $f(x)>x$ on $I$, so that $f^{n}(x) \rightarrow \infty$ on $I$, from which it follows that $f^{n}(z) \rightarrow \infty$ in the whole of $D_{1}$ by Theorem 5 . 
For any positive $\lambda$ the function $g(z)=\lambda^{-1} f(\lambda z)$ satisfies $g^{n}(z)=\lambda^{-n} f^{n}(\lambda z)$. Thus $\mathbb{E}(g)$ has the unbounded invariant component $\lambda^{-1} D_{1}$ in which $g^{n} \rightarrow \infty$. Further, $g$ has order $\frac{1}{2}$ and type $\lambda$.

\section{Proof of Theorem 1}

We quote a result closely related to the ideas of Lemma 1 and 2. It was first proved in this form by $\mathrm{H}$. Cremer [8].

LEMMA 6. If $f$ is a non-linear entire function and in a component $D$ of $\$(f)$ some subsequence of $\left(f^{n}\right)$ has a non-constant limit function, then there is a component $D_{1}$ of $\Subset(f)$ and a positive integer $p$ such that $f^{p}$ maps $D_{1}$ univalently on $D_{1}$ and for some $n_{k} \rightarrow \infty$ we have $f^{p n_{k}}(z) \rightarrow z$ in $D_{1}$. Moreover $f^{N}(D) \subset D_{1}$ for some $N$.

LEMMA 7. If $f$ is a non-linear entire function and $\mathfrak{E}(f)$ has an unbounded component $D$ in which every limit function of a subsequence of $\left(f^{n}\right)$ is finite, then the growth of $f$ is at least of order $\frac{1}{2}$, mean type.

Proof. The hypotheses ensure that $f$ is transcendental in view of III of Section 1. We suppose that the growth of $f$ is at most of order $\frac{1}{2}$, zero type.

Take a fixed point $\alpha$ in $D$. Then $\left(f^{n}(\alpha)\right)$ is bounded, for otherwise there is a sequence $n_{k}$ of integers which tends to $\infty$ and is such that $f^{n_{k}}(\alpha) \rightarrow \infty$. By replacing $n_{k}$ by one of its subsequences we can assume that $f^{n_{k}}$ is locally uniformly convergent in $D$ to a limit which can only be the infinite constant.

let $A$ be a constant such that $A>1$ and $\left|f^{n}(\alpha)\right|<A$ for all $n$. Let $\gamma$ be a path in $D$ which joins $\alpha$ to a point $\beta$ of $D$, such that the minimum modulus function

$$
m(f, r)=\min _{|z|=r}|f(z)|
$$

satisfies $m(f,|\beta|)>|\beta|^{2}$, and also $|\beta|>2 A$. This is impossible (see for example [14]) since $\left(f(z)-a_{0}-a_{1} z\right) / z^{2}$ is at most of order $\frac{1}{2}$, zero type and so has unbounded minimum modulus function.

Now $f(\gamma)$ joins $f(\alpha)$ to some point at least beyond $|z|=|\beta|$ and by induction we see that $f^{n}(\gamma)$ contains $f^{n}(\alpha)$ inside $|z|<A$ and some other point $z_{1}$ such that $\left|z_{1}\right|=|\beta|>2 A$. Then $f^{n_{k}}$ cannot have a constant limit on $\gamma$ for any subsequence $"{ }_{k}$ and the only possible limits in $D$ are non-constant.

By Lemma 6 there is a component of $\mathbb{E}(f)$ which is mapped univalently onto itself by some $f^{p}$ and the identity function $z$ is a limit of some sequence of iterates $f^{p m_{*}}$ in $D_{1}$. Since $f^{N}(D) \subset D_{1}$ for some integer $N$ it follows from the unbounded minimum modulus of $f$ that $f^{N}(D)$ and hence also $D_{1}$ are unbounded. 
Now $f^{N}(\gamma)$ contains $\alpha^{\prime}=f^{N}(\alpha)$, with $\left|\alpha^{\prime}\right|<A$, and a point $z^{\prime}$ such that $\left|z^{\prime}\right|=|\beta|>2 A$ and $f^{N}(\gamma) \subset D_{1}$. Let $\Gamma$ be the part of $f^{N}(\gamma)$ from $\alpha^{\prime}$ to the first intersection with $|z|=|\beta|$. Then $f^{j}(\Gamma)$ always contains points of modulus at least $|\beta|^{2}>2|\beta|$, provided $j \geqslant 1$, and this is true in particular for $j=p m$, $m=1,2, \ldots$ Thus it is impossible that $f^{p n_{k}}(z) \rightarrow z$ on $\Gamma$. This contradicts the result derived in the preceding paragraph, and so the growth of $f$ must exceed order $\frac{1}{2}$, zero type. Bhattacharyya [7] showed that the function $F(z)=$ $\cos \left\{\left(\varepsilon^{2} z+\frac{9}{4} \pi^{2}\right)^{1 / 2}\right\}, 0<\varepsilon<(3 \pi)^{1 / 2}$, which has order $\frac{1}{2}$, type $\varepsilon$ has an attractive fixed point at $0: F(0)=0, F^{\prime}(0)=\varepsilon^{2} /(3 \pi)$. Thus 0 belongs to a component $D$ of $\Xi(F)$ in which $F^{n}(z) \rightarrow 0$. He showed further that $D$ contains the interval $[-1, \infty)$ of the real axis and is therefore unbounded and invariant. Since all singularities of $F^{-1}$ lie over \pm 1 which are in $D$, we can continue the branch of $g=F^{-1}$ which satisfies $g(0)=0$ into any branch of $F^{-1}(z), z \in D$, along a path $\gamma$ which joins 0 to $z$ in $D$. By the complete invariance of $\mathfrak{E}$, the values $F^{-1}(\gamma)$ lie in $\mathcal{E}, g(0) \in D$ so all values $F^{-1}(z) \in D$, which is thus completely invariant.

Proof of Theorem 1. Suppose that $f$ is transcendental entire with an unbounded invariant domain $D$. Then Theorem 5 shows that either every limit function is finite or else $f^{n}(z) \rightarrow \infty$ in $D$. In the first case the result follows from Lemma 7. In the second Theorem 6 shows that there is a path approaching $\infty$ on which $|f(z)|=O\left(|z|^{k}\right)$ for some $k$ and the result follows by the minimum modulus properties of functions of growth at most order $\frac{1}{2}$, zero type. The sharpness of the rate of growth in Theorem 1 is shown both by Bhattacharyya's function $F$ quoted above, and by the function $g(z)$ described in discussing the corollary to Theorem 6. $F(z)$ also shows the sharpness of Theorem 3 of the introduction.

\section{Proof of Theorem 2}

Lemma 8 (Barry [5, p. 473, Theorem 5]). Let $f(z)$ be a non-constant entire function which, for $p>1$, satisfies

$$
\log M(r, f)=O(\log r)^{p} \quad(r \rightarrow \infty) .
$$

If $\varepsilon, \eta$ and $C$ are constants such that $\eta>\varepsilon>0$ and $C>0$ and

$$
E=E\left\{r ; \log m(r, f) \leqslant \log M(r, f)-C(\log r)^{p-2+\eta}\right\},
$$

then

$$
\int_{E} d(\log r)^{\eta-e}<\infty
$$

Here $m(r, f)=\min _{|z|=r}|f(z)|$. 
LEMMA 9. If the transcendental entire function $f$ satisfies

$$
\log M(r, f)=O(\log r)^{p} \quad(r \rightarrow \infty)
$$

where $1<p<3$, then there exists a sequence $R_{n} \rightarrow \infty$, such that

(i) $M\left(R_{n}, f\right)=R_{n+1}, n=1,2, \ldots$,

(ii) $s_{n}=\log R_{n}>16^{n} \log 16$,

(iii) there exists $\rho_{n}$ which satisfies both

$$
R_{n}<\rho_{n}<R_{n}^{2+n^{-1}}
$$

and

$$
m\left(\rho_{n}, f\right)>R_{n+1}^{2+(n+1)^{-1}}
$$

for all sufficiently large $n$.

Proof. Since $f$ is transcendental there exists $r_{0}$ such that

$$
M(r)=M(r, f)>r^{16}, \quad r>r_{0} .
$$

Take $R_{1}>\operatorname{Max}\left(16^{16}, r_{0}\right)$. Then defining $R_{n}$ by (i) we have

$$
R_{n+1}=M\left(R_{n}\right)>R_{n}^{16}>R_{n},
$$

whence (ii) follows by induction.

Since $p<3$ we may choose $\eta>0$ such that $p+\eta<3$ and $\varepsilon$ so that $\varepsilon>0$ and $\lambda=\eta-\varepsilon>0$. Choose $C=1$. In Lemma 8 the measure condition on $E$ shows that for sufficiently large $r$ the interval $\left(r_{1}, r\right)$ must contain a point in the complement of $E$, provided that $r_{1}$ is defined by

$$
(\log r)^{\lambda}-\left(\log r_{1}\right)^{\lambda}=1 \text {. }
$$

Then

$$
\log r_{1}=\log r-\lambda^{-1}(\log r)^{1-\lambda}+O(\log r)^{1-2 \lambda} \quad \text { as } r \rightarrow \infty,
$$

so

$$
\log r_{1}>\log r-2 \lambda^{-1}(\log r)^{1-\lambda}>\frac{1}{2} \log r, \quad r>r_{2} .
$$

By Lemma 8 there is $\rho=\rho(r)$ in $\left(r_{1}, r\right)$ such that $m(\rho)=m(\rho, f)$ satisfies

$$
\log m(\rho)>\log M(\rho)-(\log \rho)^{\mu},
$$

where $\mu=p-2+\eta<1$. For $r=R_{n}^{2+n^{-1}}, n$ sufficiently large, we can find such a $\rho(r)$ and we call this $\rho_{n}$. By (2) we have

$$
R_{n}^{2+n^{-1}}>\rho_{n}>R_{n}^{1+\frac{1}{2} n^{-1}}>R_{n},
$$

which is the first part of (iii). 
Also by (3)

$$
\log m\left(\rho_{n}\right)>\log M\left\{r_{1}\left(R_{n}^{2+n^{-1}}\right)\right\}-\left\{\left(2+\frac{1}{n}\right) \log R_{n}\right\}^{\mu} .
$$

Putting $V(s)=\log M\left(e^{s}\right), s_{n}=\log R_{n}$ we have by (2)

$$
\begin{aligned}
\log r_{1}\left(R_{n}^{2+n^{-1}}\right) & >\left(2+\frac{1}{n}\right) s_{n}-2 \lambda^{-1}\left\{\left(2+\frac{1}{n}\right) s_{n}\right\}^{1-\lambda} \\
& =\left(2+\frac{2}{2 n+1}\right) s_{n}+\frac{s_{n}}{n(2 n+1)}-\frac{2}{\lambda}\left\{\left(2+\frac{1}{n}\right) s_{n}\right\}^{1-\lambda} \\
& >\left(2+\frac{2}{2 n+1}\right) s_{n}
\end{aligned}
$$

for large $n$ by (ii). Since $V$ is an increasing function (4) yields

$$
\log m\left(\rho_{n}\right)>V\left\{\left(2+\frac{2}{2 n+1}\right) s_{n}\right\}-\left\{\left(2+\frac{1}{n}\right) s_{n}\right\}^{\mu} .
$$

Since $V$ is a convex function of $s$ we have

$$
\begin{aligned}
V\left\{\left(2+\frac{2}{2 n+1}\right) s_{n}\right\}> & \left(2+\frac{2}{2 n+1}\right) V\left(s_{n}\right)-\left(1+\frac{2}{2 n+1}\right) V(0) \\
> & \left(2+\frac{1}{n+1}\right) V\left(s_{n}\right)+\frac{s_{n}}{(2 n+1)(n+1)} \\
& -\left(1+\frac{2}{2 n+1}\right) V(0)
\end{aligned}
$$

for large $n$, since $V(s)>16 s>s$ for $s>\log r_{0}$. By combining the above inequality with (ii), we see that the right hand side of (5) is at least $\left(2+(n+1)^{-1}\right) V\left(s_{n}\right)$ for large $n$. But this is equivalent to

$$
m\left(\rho_{n}, f\right)>\left\{M\left(R_{n}, f\right)\right\}^{2+(n+1)^{-1}}=R_{n+1}^{2+(n+1)^{-1}} .
$$

Proof of THE Theorem. Suppose that $f$ satisfies the hypotheses of the theorem and also that $\sqrt{5}(f)$ has an unbounded component $D$. We may assume without loss of generality that 0,1 belong to $\mathfrak{F}(f)$. Construct sequences $R_{n}$ and $\rho_{n}$ as in Lemma 9 and let $n_{1}$ be such that conditions (i), (ii), (iii) hold for $n>n_{1}$. Take a fixed $n>n_{1}$. Then $D$ meets the circles $\gamma:|z|=R_{n}$ and $\gamma^{\prime}:|z|=$ $R_{n+1}^{\left(2+(n+1)^{-1}\right)}$ so there is a path $C$ in $D$ which joins a point of $\gamma$ to a point of $\gamma^{\prime}$ and contains a point $\rho_{n+1} e^{i \varphi_{n+1}}$.

Now $f(D)$ is an unbounded component of $\mathbb{E}(f)$ and contains the path $f(C)$ which joins a point $w_{n}=f\left(R_{n} e^{i \theta_{n}}\right)$, say, in $\left|w_{n}\right| \leqslant R_{n+1}$ to a point $w_{n}^{\prime}=$ $f\left(\rho_{n+1} e^{i \varphi_{n+1}}\right)$ with

$$
\left|w_{n}^{\prime}\right|>R_{n+2}^{2+(n+1)^{-1}} \text {. }
$$

Hence $f(C)$ contains an arc which joins $|z|=R_{n+1}$ to $|z|=R_{n+2}^{2+(n+2)^{-1}}$ in $f(D)$. Continuing by induction we see that $f^{k}(D)$ is an unbounded component of $\mathbb{E}(f)$ 
containing an arc of $f^{k}(C)$ which joins $|z|=R_{n+k}$ to

$$
|z|=R_{n+k+1}^{2+(n+k+1)^{-1}}
$$

Thus on $C$ the function $f^{k}$ takes a value of modulus at least $R_{n+k}$. Since $R_{n} \rightarrow \infty$ as $n \rightarrow \infty$ we see that the only possible limit function of $\left(f^{k}\right)$ is $\infty$ and so $f^{k}(z) \rightarrow \infty$ locally uniformly in $D$ as $k \rightarrow \infty$.

Since all $f^{k}$ omit 0,1 in $D$ and since $\left|f^{k}\right|>1$ on $C$ for, say, $k>k_{0}$, Lemma 5 shows that there are constants $A, B$ such that

$$
\left|f^{k}\left(z^{\prime}\right)\right|<A\left|f^{k}(z)\right|^{B}, \quad z, z^{\prime} \in C, k>k_{0} .
$$

In particular

$$
R_{n+k+1}<A R_{n+k}^{B}, \quad k>k_{0}
$$

i.e.

$$
M\left(R_{j}\right)<A R_{j}^{B}, \quad j>n+k_{0} .
$$

Since $R_{j} \rightarrow \infty$ this contradicts the assumption that $f$ is transcendental and the theorem is proved.

\section{References}

[1] Baker, I. N., 'Repulsive fixpoints of entire functions', Math. Z. 104 (1968) 252-256.

[2] _ 'Completely invariant domains of entire functions', Math. essays dedicated to A.J. MacIntyre (Ohio University Press, Athens, Ohio, 1970).

[3] _ 'Limit functions and sets of non-normality in iteration theory', Ann. Acad. Sci. Fenn. Ser. A I, 467, 1-11 (1970).

[4] _ 'The domains of normality of an entire function', Ann. Acad. Sci. Fenn. Ser A I, 1, (1975) 277-283.

[5] Barry, P. D., 'The minimum modulus of small integral and subharmonic functions', Proc. London Math. Soc. (3) 12, (1962) 445-495.

[6] Bhattacharyya, P., 'On the domain of normality of an attractive fixpoint'. Trans. Amer. Math. Soc. 153, (1971) 89-98.

[7] __ Iteration of analytic functions (Ph.D. thesis, University of London, 1969).

[8] Cremer, H., 'Uber die Schrödersche Funktionalgleichung u.s.w.', Ber. Verh. Sächsische Akad. Leipzig 84, (1932) 291-324.

[9] Denjoy, A., 'Sur l'itération des fonctions analytiques', C. R. Acad. Sci. Paris Sér A-B 182, (1926) 275-257.

[10] Fatou, P., 'Sur les équations fonctionelles', Bull. Soc. Math. France 47, (1919) 161-271; 48, (1920) 33-94 and 208-314.

[11] _ 'Sur l'itération des fonctions transcendantes entières', Acta Math. 47, (1926) 337370.

[12] Julia, G., 'Mémoire sur l'itération des fonctions rationelles', J. Math. Pures Appl. (8) 1, (1918) 47-245.

[13] Hayman, W. K., Meromorphic functions (The Clarendon Press. Oxford, 1964).

[14] Heins, M., 'Entire functions with bounded minimum modulus; subharmonic function analogues', Ann. of Math. (2) 49, (1948) 200-213. 
[15] Rüssman, H., 'Über die Iteration analytischer Funktionen', J. Math. Mech. 17, (1967) 523-532.

[16] Whyburn, G. T., Analytic Topology (Am. Math. Soc. Colloquium Pub. Providence, U.S.A. (1942)).

[17] Wolff, J., 'Sur l'itération des fonctions bornées', C. R. Acad. Sci. Paris Sér A-B 182, (1926) 200-201.

\section{Department of Mathematics}

Imperial College

London SW 7 2AZ. 